\title{
Pillen, Kapseln und Tabletten
}

\author{
B. Gurtner
}

Der weltbekannte Kardiologe und Friedensnobelpreisträger Bernard Lown berichtet in seinen Lebenserinnerungen «Die verlorene Kunst des Heilens» über einen schwierig zu führenden Patienten, bei dem die Therapie seines tachykarden Vorhofflimmerns wirkungslos blieb. Es stellte sich heraus, dass der ängstliche Mann die Chinidintabletten zu einem Pulver zerdrückt und Brotkrumen beigemischt hatte, die er an Tauben verfutterte, welche auf der Brüstung vor dem Fenster des Krankenhauses nisteten. Eine Taube habe den Köder aufgepickt und sei kurz darauf tot vornübergefallen, was für den Patienten der Beweis war, dass den Ärzten und ihren Verordnungen nicht zu trauen sei, weshalb er sich auch nicht weiter daran hielt.

$\mathrm{Zu}$ meinen Kindheitseindrücken gehört ein wohlbeleibter rotwangiger Onkel, dem der Hausarzt allerlei farbige Pillen gegen hohen Blutdruck, Zuckerkrankheit und böse Beine verschrieben hatte, der es aber immer wieder fertigbrachte, die von der Tante liebevoll bereitgelegten Medikamente verschwinden zu lassen, bevor sie die Einnahme überwachen konnte. Mit listigem Augenzwinkern raffte er die Kapseln und Kügelchen mit der linken Hand zusammen und verschwand auf der Toilette, in der nach einem kurzen Zwischenhalt der rauschende Sturzbach der Wasserspülung zu hören war. Er zeigte mir auch heimlich, dass man Pillen in Blumenvasen oder in der Erde von Topfpflanzen verstecken kann, und behauptete, die Blüten blieben deshalb länger frisch.

Viele Jahre später erzählte ein Spitalgärtner beim Pausenkaffee, dass er auf dem Rasen und in den Blumenbeeten entlang des Bettenhauses immer wieder Medikamente finde. Der bodenständige Fachmann meinte, dass es den Pflanzen ganz bestimmt nicht guttue, den Patienten vielleicht aber schon, weil damit ja auch viele Nebenwirkungen wegfielen. Darauf wurde ein Unterassistent ausgeschickt für eine Feldstudie, bei der in kurzer Zeit zahlreiche Pillen, Kapseln und Tabletten eingesammelt werden konnten. Da wurde buchstäblich eine Menge Geld aus den Fenstern der Krankenzimmer geworfen.
Nach verschiedenen Schätzungen, die allerdings auf Dunkelziffern beruhen, werden mindestens $25 \%$ aller abgegebenen Medikamente nicht eingenommen, irgendwie entsorgt oder in den Hausapotheken für allfälligen späteren Bedarf jahrzehntelang gelagert. In diesen privaten pharmazeutischen Museen vermodern Präparate von Herstellern, die es wegen Fusionen oder Betriebsschliessungen bereits nicht mehr gibt. Auch die Wirkstoffe haben sich längst zersetzt oder verflüchtigt. Doch die Salbe, welche Grossvaters Knie so gut geholfen hat, wird aufbewahrt, bis die Tube bricht und das ranzige Fett ausläuft. Böse Zungen behaupten, dass auch in Hausapotheken von Medizinalpersonen uralte Pakkungen lägen. In Arzttaschen und Erste-HilfeKoffern seien Ampullen mit merkwürdiger Farbe oder trübem Bodensatz zu finden, deren Verfalldaten bereits am Ende des letzten Jahrhunderts unbemerkt verstrichen sind.

Die jährlichen Heilmittelkosten in der Schweiz betragen bald 4 Milliarden Franken. Die Annahme, dass davon etwa ein Viertel wegen Unverträglichkeiten, mangelnder Compliance oder routinemässiger Abgabe zu grosser Packungen verschwendet werde, ist glaubhaft und müsste aufhorchen lassen. Wegen der vorherrschenden Geschäftstüchtigkeit der Produzenten und Verkäufer sowie der unüberwindbaren Nachlässigkeit der Konsumenten besteht aber wenig Hoffnung auf baldigen Umschwung. Lobenswerte Bemühungen, individuell kleinere Pakkungen abzugeben und die Menge der täglich einzunehmenden Präparate auf wenige Schluckeinheiten (SE/d) einzuschränken, haben bisher keine breite Resonanz gefunden. So bleiben wir Schweizer statistisch gesehen führend im Tablettenschlucken, vielleicht aber sind wir nur Weltmeister im Pillenverteilen und Pillenverstecken sowie im bewussten Verdrängen der immensen Verluste, die daraus für die Kostenträger entstehen, dem Bruttosozialprodukt aber offenbar zunutze sind.

- Bernard Lown. Die verlorene Kunst des Heilens. Berlin: Suhrkamp Taschenbuchverlag; 2004. Originalausgabe: The Lost Art of Healing. New York: Houghton Miffin; 1996. 